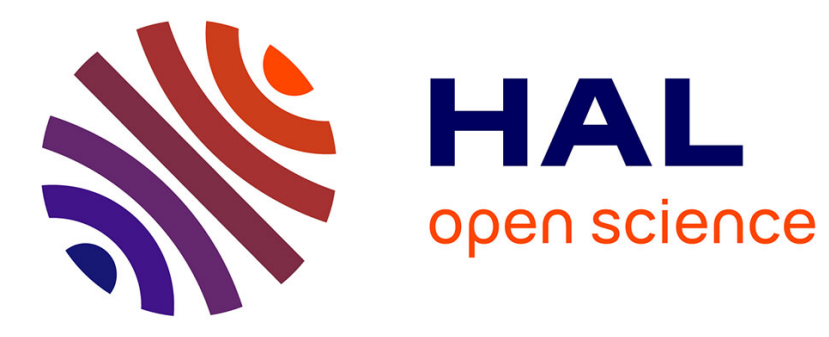

\title{
Probing Membrane Protein Insertion into Lipid Bilayers by Solid-State NMR
}

\author{
Eszter Najbauer, Kumar Tekwani Movellan, Tobias Schubeis, Tom S \\ Schwarzer, Kathrin Castiglione, Karin Giller, Guido Pintacuda, Stefan Becker, \\ Loren B Andreas
}

\section{To cite this version:}

Eszter Najbauer, Kumar Tekwani Movellan, Tobias Schubeis, Tom S Schwarzer, Kathrin Castiglione, et al.. Probing Membrane Protein Insertion into Lipid Bilayers by Solid-State NMR. ChemPhysChem, 2018, BioNMR Spectroscopy, 20 (2), pp.302-310. 10.1002/cphc.201800793 . hal-02386264

\section{HAL Id: hal-02386264 https://hal.science/hal-02386264}

Submitted on 30 Nov 2021

HAL is a multi-disciplinary open access archive for the deposit and dissemination of scientific research documents, whether they are published or not. The documents may come from teaching and research institutions in France or abroad, or from public or private research centers.
L'archive ouverte pluridisciplinaire HAL, est destinée au dépôt et à la diffusion de documents scientifiques de niveau recherche, publiés ou non, émanant des établissements d'enseignement et de recherche français ou étrangers, des laboratoires publics ou privés. 


\title{
Probing Membrane Protein Insertion into Lipid Bilayers by Solid-State NMR
}

Eszter E. Najbauer ${ }^{3}$, Kumar Tekwani Movellan ${ }^{3}$, Tobias Schubeis ${ }^{1}$, Tom Schwarzer ${ }^{2}$, Kathrin Castiglione $^{2}$, Karin Giller ${ }^{3}$, Guido Pintacuda ${ }^{1}$, Stefan Becker ${ }^{3}$, Loren B. Andreas ${ }^{1,3}$

1) Centre de RMN à Très Hauts Champs, Institut des Sciences Analytiques, UMR 5280/CNRS, ENS Lyon, UCB Lyon 1, Université de Lyon, Villeurbanne, France

2) Institute of Bioprocess Engineering, Friedrich-Alexander-Universität Erlangen-Nürnberg, PaulGordan-Straße 3, 91052 Erlangen, Germany

3) Department of NMR based Structural Biology, Max Planck Institute for Biophysical Chemistry, Am Fassberg 11, 37077 Göttingen, Germany

\begin{abstract}
Determination of the environment surrounding a protein is often key to understanding its function and can also be used to infer the structural properties of the protein. By using proton-detected solidstate NMR, we show that reduced spin diffusion within the protein under conditions of fast magicangle spinning, high magnetic field, and sample deuteration allows the efficient measurement of site-specific exposure to mobile water and lipids. We demonstrate this site specificity on two membrane proteins, the human voltage dependent anion channel, and the alkane transporter AlkL from Pseudomonas putida. Transfer from lipids is observed selectively in the membrane spanning region, and an average lipid-protein transfer rate of $6 \mathrm{~s}^{-1}$ was determined for residues protected from exchange. Transfer within the protein, as tracked in the ${ }^{15} \mathrm{~N}-{ }^{-1} \mathrm{H}$ 2D plane, was estimated from initial rates and found to be in a similar range of about 8 to $15 \mathrm{~s}^{-1}$ for several resolved residues, explaining the site specificity.

Cellular membranes are essential for maintaining homeostasis in an organism by separating cellular processes and controlling transmembrane signaling and the flow of metabolites. These processes are regulated by various proteins, and invariably involve integral membrane proteins that span the lipid bilayer as beta barrels in mitochondria and prokaryotes, or as alpha helices in both eukaryotes and prokaryotes. Since the lipid bilayer may be necessary for maintaining membrane protein structure and function, it is crucial to develop methods for the study of membrane proteins in such nearnative environments..$^{1-3}$ A key advantage of magic-angle spinning (MAS) NMR in this context, is the ability to study membrane proteins prepared in planar lipid bilayers at atomic resolution for investigation of structure and dynamics. ${ }^{4-6}$ In addition, the proximity of mobile species such as lipids and water, that are difficult if not impossible to capture via cryo-electron microscopy or X-ray crystallography, can be probed with MAS NMR. In this way, it is possible to investigate solvent accessibility $^{7-9}$ or membrane association. ${ }^{10}$
\end{abstract}

The environment of the protein surface can be probed by magnetization transfer between the protein and components of its environment, which is most commonly water, but in the case of membrane proteins is also lipids. While there is a choice of NMR active nucleus for the lipids, the more universal approach, and indeed the approach most commonly implemented in literature is to transfer magnetization by longitudinal proton-proton mixing. At physiological temperatures, water rapidly diffuses, which results in long proton T2 relaxation times. In the case of lipids, anisotropic diffusion 
results in proton $\mathrm{T} 2$ times of tens of ms, ${ }^{11}$ which is still at least an order of magnitude longer than for the protein under moderate spinning of $\sim 10 \mathrm{kHz}$. This allows the water or lipid signals to be selected based on differential relaxation times ${ }^{12-14}$ or to distinguish multiple water pools with distinct relaxation properties within the same sample.,

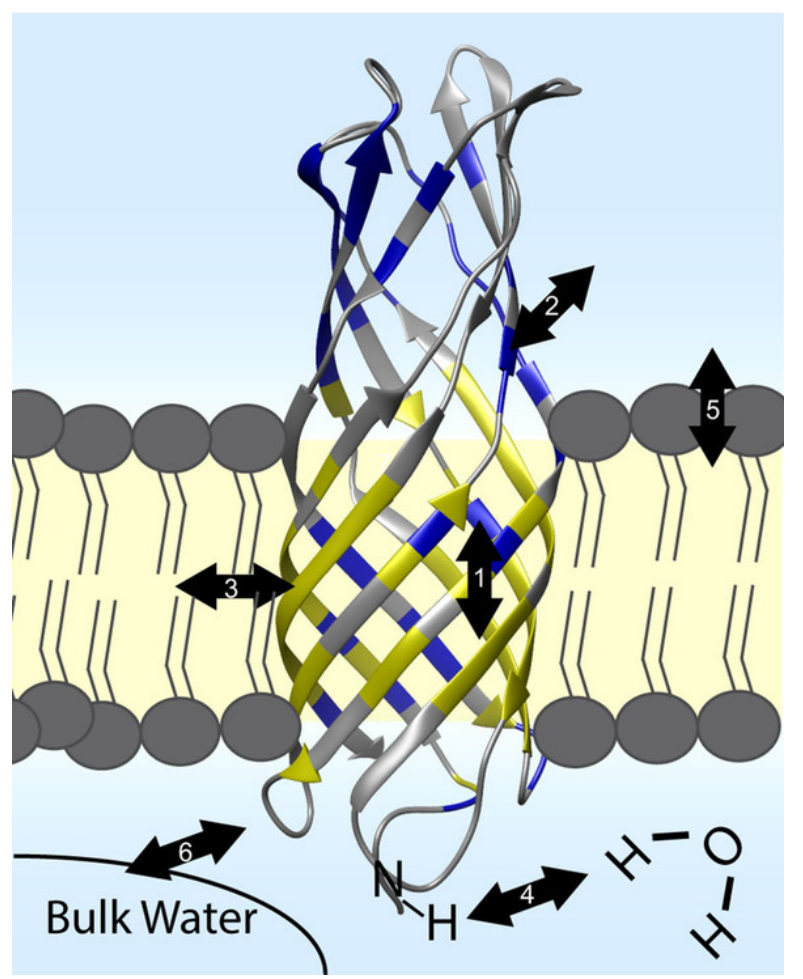

Figure 1: Schematic representation of longitudinal magnetization transfer in a lipid bilayer sample. 1-6 shows transfer between 1, protein and protein, 2, water and protein, 3, lipid and protein, 4, water and protein by chemical exchange, 5, lipid and water, and 6, bulk and associated water.

Different primary mechanisms for water-protein magnetization transfer are possible under this scheme, including the nuclear Overhauser effect (NOE), chemical exchange, and dipolar mediated spin diffusion. Since the NOE mechanism relies on cross relaxation of dipolar coupled spins, and requires correlated motion of the two coupled spins, it is not usually considered in solid-state NMR. However, Zilm and coworkers showed that water-protein transfer was consistent with NOE relaxation in the solid state for a deuterated protein using HN detection. ${ }^{16}$ The conclusion was drawn by comparison of magnetization transfer rates with and without recoupling, and in the rotating frame. Under different experimental conditions, Böckmann and coworkers found chemical exchange to be an important factor governing the buildup, which in their case was detected through cross polarization to carbon resonances in protein microcrystals and fibrils. ${ }^{8,17}$ The fact that these carbon resonances are closer to exchangeable $\mathrm{NH}$ - and $\mathrm{OH}$-protons in the sidechains may in part explain the differing contributions of NOE and chemical exchange in the two studies. In addition, details of the sample condition may also play a role, since the rate of chemical exchange can be reduced by decreasing the sample temperature and changing the $\mathrm{pH}$. For site specificity, the possibility for dipolar mediated spin diffusion complicates the picture as compared with membrane protein studies in solution. ${ }^{18}$ Spin diffusion in solids is typically mediated by dipolar couplings, although diffusion of magnetization also occurs through relayed NOE transfers at long mixing times. In general, magnetization transfer within the protein can occur through spin diffusion and NOE, while transfer between the protein and the surrounding mobile water or lipids can take place through NOE. For water, chemical exchange is also possible. For phosphocholine lipids, the exchange pathway is not possible, as the lipid molecules do not have exchangeable protons, and 
dipolar spin diffusion is reduced by considerable anisotropic motion. A summary of the magnetization transfer pathways is shown in Figure 1.

At low MAS rates and in fully protonated proteins, dipolar mediated spin diffusion is rapid, with efficient proton-proton transfer occurring in hundreds of microseconds. ${ }^{19}$ Typical NOE mixing times are orders of magnitude longer, since the NOE rates are lower. The result is that since the waterprotein transfer is rate limiting, water magnetization is passed onto the protein primarily via fast exchanging groups and NOE, then spread quickly through the protein such that site specificity is obscured..$^{14}$ Nevertheless, broader changes in the buildup of magnetization can be detected even at moderate MAS rates, for example, the reduction in exposed surface due to drug binding was evident in the influenza M2 protein. ${ }^{20}$ At high deuteration levels, high magnetic fields, and high spinning frequencies, the proton spectrum of the protein narrows as dipolar couplings are averaged at fast MAS, ${ }^{21}$ and the contribution of spin diffusion to magnetization transfer is reduced dramatically, allowing site resolved measurement of water proximity. ${ }^{8,16,17}$ We propose that this reduction in intraprotein transfer rates could be ideal for probing site specific lipid exposure, since the NOE transfer from the mobile lipid acyl chains could become similar to and potentially faster than the intraprotein transfer. Such site specificity would be consistent with previous measurement of specific water-protein transfer under similar conditions of deuteration and MAS rates, in which the mechanism was found to be mediated by chemical exchange. ${ }^{8,17}$ In the case of transfer from the mobile aliphatic lipid chains, chemical exchange is not possible, and we therefore are left with NOE as the dominant mechanism, although there may also be some residual transfer through spin diffusion.

Development of faster MAS frequencies, now applied in commercial probes at up to $111 \mathrm{kHz}$, have opened up new possibilities due to improved sensitivity, improved coherence lifetimes, and access to protons as the detection nucleus. ${ }^{22-29}$ Using $1 \mathrm{H}$ detection, sensitivity is increased 8 and 32-fold on a per mole basis, with respect to $13 \mathrm{C}$ and $15 \mathrm{~N}$-detection. This assumes no changes in linewidth, which is reasonable with deuteration ${ }^{29}$ and fast spinning. While fractional deuteration or smaller rotors capable of faster spinning reduces this gain somewhat, there is still an overall benefit in sensitivity with proton detection at $60 \mathrm{kHz}$ MAS. ${ }^{30}$ Furthermore, the accessibility of a well-resolved proton dimension decreases degeneracy of chemical shifts and makes unambiguous assignments possible even on large proteins.

Early implementations for determining water accessibility rely on establishing polarization selectively on water, then transferring this polarization to the protein. Water can be selected using a T2 filter $^{31,32}$ or a selective excitation pulse. ${ }^{12}$ The T2 filter uses a Hahn echo to select mobile molecules, as they can have much longer coherence times than rigid species such as protein. Subsequently, during a longitudinal mixing period, magnetization is transferred to the protein, and is then detected on $13 \mathrm{C}$ or $15 \mathrm{~N}$. In a bilayer, lipids are sufficiently mobile that the above mentioned strategies used for selection of water can also be applied to them. ${ }^{14}$ Alternatively, a spectral dimension can be used to distinguish mobile components from resonances of the protein. ${ }^{8,10,16,17}$ This approach is effective for faster MAS rates, where there is less difference in protein and water or lipid $\mathrm{T} 2$ relaxation times.

Here we apply fast MAS (55 kHz) in combination with perdeuteration and proton exchange for proton-detected measurement of water and lipids in contact with the protein. These conditions allow site-specificity within about $3 \AA$ and determination of lipid insertion depth for membrane proteins, which is critical for evaluating the position of a membrane protein in its native environment, and thus for gaining a deeper understanding of its structural, functional, and evolutionary features. We demonstrate the applicability of the method on two integral membrane proteins, AlkL from Pseudomonas putida, and the human voltage-dependent anion channel (hVDAC). Both proteins fold as membrane spanning beta barrels, with both lipid and water exposed surfaces. 


\section{Materials and Methods}

2H,13C,15N-labeled AlkL was prepared as previously described. ${ }^{33,34}$ The bilayer sample was reconstituted by addition of lipids to a 0.5 lipid to protein weight ratio and removal of detergent by dialysis. 2D crystalline 2H, 13C, 15N-labeled hVDAC was prepared using established expression and refolding protocols.35-37 Preparation of 2D crystals was carried out using the protocol modified by Eddy et al.38 from the original protocol published by Dolder et al. ${ }^{39}$

NMR experiments were performed on approximately $1 \mathrm{mg}$ of sample packed into a $1.3 \mathrm{~mm}$ Bruker MAS rotor. All experiments were acquired on an $800 \mathrm{MHz}$ Bruker Avance III spectrometer at a magnetic field of $19 \mathrm{~T}$, and spinning at $55 \mathrm{kHz}$. The temperature of cooling gas was set to $250 \mathrm{~K}$, which results in an estimated sample temperature of $20^{\circ} \mathrm{C}$. The $\mathrm{HhNH}$ and hNHH spectra were recorded by averaging 2 scans per point. Acquisition time in the direct dimension was $10 \mathrm{~ms}$, in the indirect dimensions $22 \mathrm{~ms}$ on $15 \mathrm{~N}$ and $3.5 \mathrm{~ms}(\mathrm{HhNH})$ or $4.0 \mathrm{~ms}(\mathrm{hNHH})$ on $1 \mathrm{H}$. For $1 \mathrm{H}$ to $15 \mathrm{~N} \mathrm{CP}$ a contact time of $600 \mu \mathrm{s}$ was used, with a linear ramp from 78 to $98 \mathrm{kHz}$ on $1 \mathrm{H}$ and constant irradiation of $15 \mathrm{~N}$ at $36.8 \mathrm{kHz} .1 \mathrm{H}$ and $15 \mathrm{~N}$ RF field levels were determined from hard pulse calibrations. Cross-relaxation rates were determined from 3D spectra recorded with mixing times of $0,5,10,15,25,50,75,100,150$, and $300 \mathrm{~ms}$. A mixing time of $50 \mathrm{~ms}$ was chosen for the 4D spectra. For the HhnCANH spectra, 16 scans per point were averaged together and non-uniform sampling (NUS) was applied, selecting $1.98 \%$ of the points. The sampling schedule was generated by Topspin 3.5, and the spectrum reconstructed using MDD in Topspin. The indirect acquisition times were $5.3 \mathrm{~ms}$ for $1 \mathrm{H}, 10 \mathrm{~ms}$ for $13 \mathrm{C}$ and $22 \mathrm{~ms}$ for $15 \mathrm{~N}$. All experiments used waltz-16 heteronuclear decoupling at $10 \mathrm{kHz}$ on both $13 \mathrm{C}$ and $15 \mathrm{~N}$. Protons were decoupled using $7 \mathrm{kHz}$ TPPM decoupling during indirect acquisition times. The NOE mixing time was $50 \mathrm{~ms}$. The recycle delay was 0.8 seconds. This resulted in a total experiment time of 6 to 7 hours for each mixing point of the HhNH, 7 to 8 hours for each mixing point of the hNHH, and 8 days for the 4D HhnCANH.

Spectra were processed in Topspin 3.5 (Bruker) and analyzed in Sparky. ${ }^{40}$

\section{Results and Discussion}

Figure 2 shows the pulse sequences used to record 3- and 4-dimensional, z-mixing based protondetected spectra for determining site-resolved water and lipid proximities in integral membrane proteins. Figure 2A shows the 3-dimensional $\mathrm{HhNH}$ pulse sequence, which is only slightly modified from the hNHH sequence of Zilm and coworkers16 by placing the z-mixing at the beginning rather than the end of the sequence. Proton excitation is followed by an indirect evolution period to encode the frequencies of water, lipids, and protein. Next, longitudinal mixing allows proton-proton transfer between mobile small molecules and the protein, via exchange and NOE. Magnetization is then transferred to $15 \mathrm{~N}$, evolved, and transferred back to proton, where it is then detected. The outand-back transfer to $15 \mathrm{~N}$ uses two short cross-polarization (CP) steps to ensure a selective transfer. The 3D experiment can be extended to 4 dimensions by including an additional transfer to CA. This HhnCANH sequence (Figure 2B) proves useful when the HN spectrum is not sufficient to resolve signals from all residues. Similar in concept to the 3D, the out-and-back scheme in the 4D also directs transfer within one residue. The more sensitive HhCANH transfer scheme would allow longer range transfer during the $1 \mathrm{H}-13 \mathrm{C}$ transfer step. Two different $\mathrm{N}-\mathrm{C}-\mathrm{N}$ out-and-back transfer schemes were considered, based on either $\mathrm{CP}$ (Figure 2B) or transferred echo double resonance (TEDOR, Figure 2C). ${ }^{41}$ Similar to recent observations, the TEDOR transfer was found to be more efficient. ${ }^{42}$ Omitting $1 \mathrm{H}-1 \mathrm{H}$ mixing, we found the CP based sequence to perform with $14 \%$ efficiency, as compared with $20 \%$ using TEDOR, both with respect to the hNH sequence. 

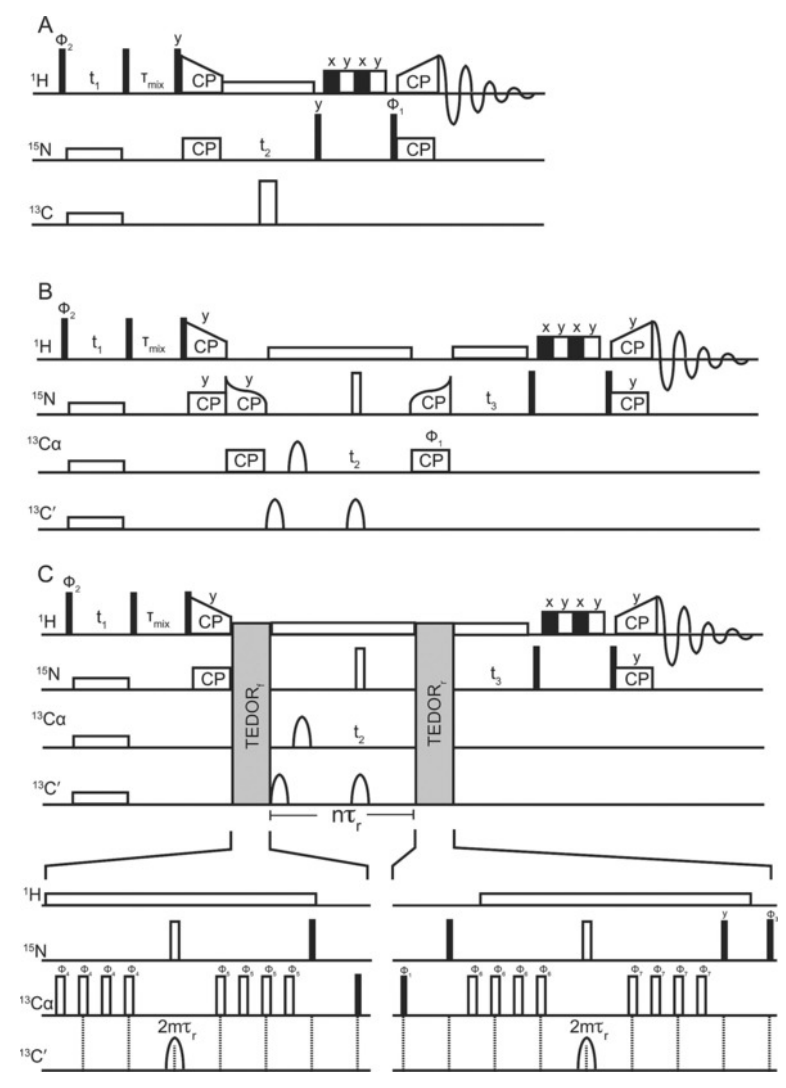

Figure 2: Pulse sequences used to record site resolved water and lipid proximity in membrane proteins. In A, the 3D HhNH sequence. In B, the CP based HhnCANH sequence. In c, the TEDOR based HhnCANH sequence. Solid and empty rectangles represent 90 and 180 degree pulses, respectively. Empty half-ovals represent selective 180 degree pulses, Q3 pulses ${ }^{43}$ in our implementation. Tangent ramped pulses were used on the $15 \mathrm{~N}$ channel for 13C15N CP transfer. A selective pulse on the carbon channel during TEDOR prevents transfer to the carbonyl. $1.45 \mathrm{~ms}$ TEDOR transfer was used. Proton decoupling of 5 to $15 \mathrm{kHz}$ WALTZ or swept TPPM decoupling was used during TEDOR, while $12 \mathrm{kHz}$ swept TPPM was used during evolution periods. WALTZ decoupling on the carbon and nitrogen channels was set to $10 \mathrm{kHz}$. Rotor synchronization is indicated by vertical dashed lines.

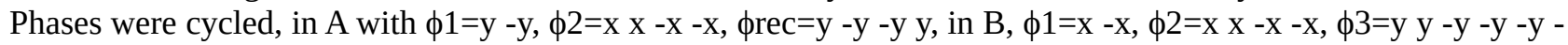

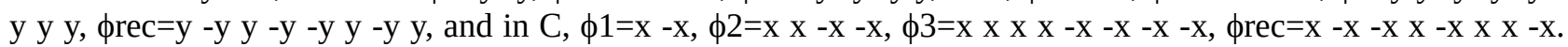
REDOR pulses on the carbon channel in $C$ followed the $x y-16$ scheme. All other pulses were applied with phase $\mathrm{X}$.

In all three pulse sequences, the $1 \mathrm{H}-1 \mathrm{H}$ mixing takes place at the beginning, preceded by evolution in an indirect proton dimension. This carries the advantage that the indirect sampling can be limited to reduce the overall data acquisition time. This results in lower resolution in the indirect proton dimension, which is sufficient as this dimension serves only to distinguish between lipids, water and protein resonances. In a perdeuterated protein, the aliphatic proton resonances of the protein are suppressed, and lipid peaks of DMPC appear isolated at about 0.9, 1.3, and $3.3 \mathrm{ppm}$ for acyl chain $\mathrm{CH} 3$, acyl chain $\mathrm{CH} 2$ and choline $\mathrm{CH} 3$ moieties, respectively. The directly detected proton dimension, used for distinguishing between protein residues, can be sampled out as far as needed without additional cost in measurement time. Secondly, placing the NOE mixing directly before acquisition instead would allow water and lipid magnetization to build up during the mixing time, which would result in problems with water suppression.

Figure 3 shows selected strips of the 3D spectra on AlkL and hVDAC at resolved amide sites, as well as the corresponding buildup of magnetization transferred from either lipid or water. Magnetization buildup increases on water and lipids until about $100 \mathrm{~ms}$, however at the longer mixing times, magnetization may also be transferred to nearby protein residues, resulting in the loss of the experiment's specificity. Even without a detailed fitting of relaxation rates, it can be seen that the buildup of the inter-residue cross-peaks occurs with a similar rate as compared to lipid-protein 


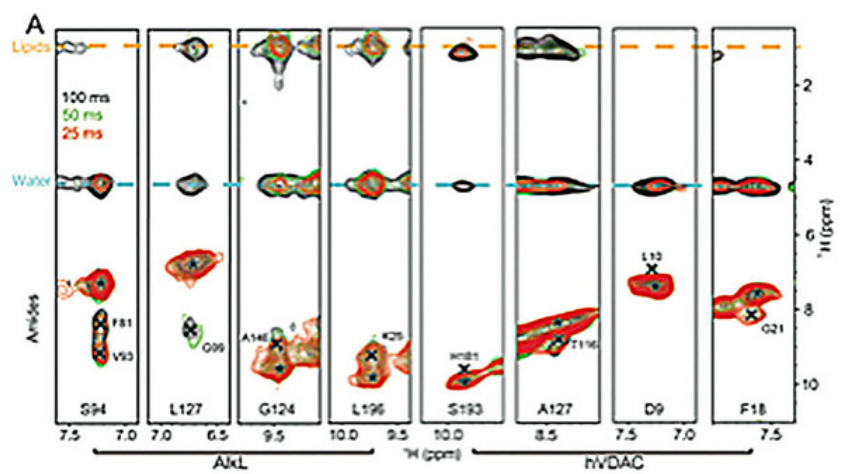

B
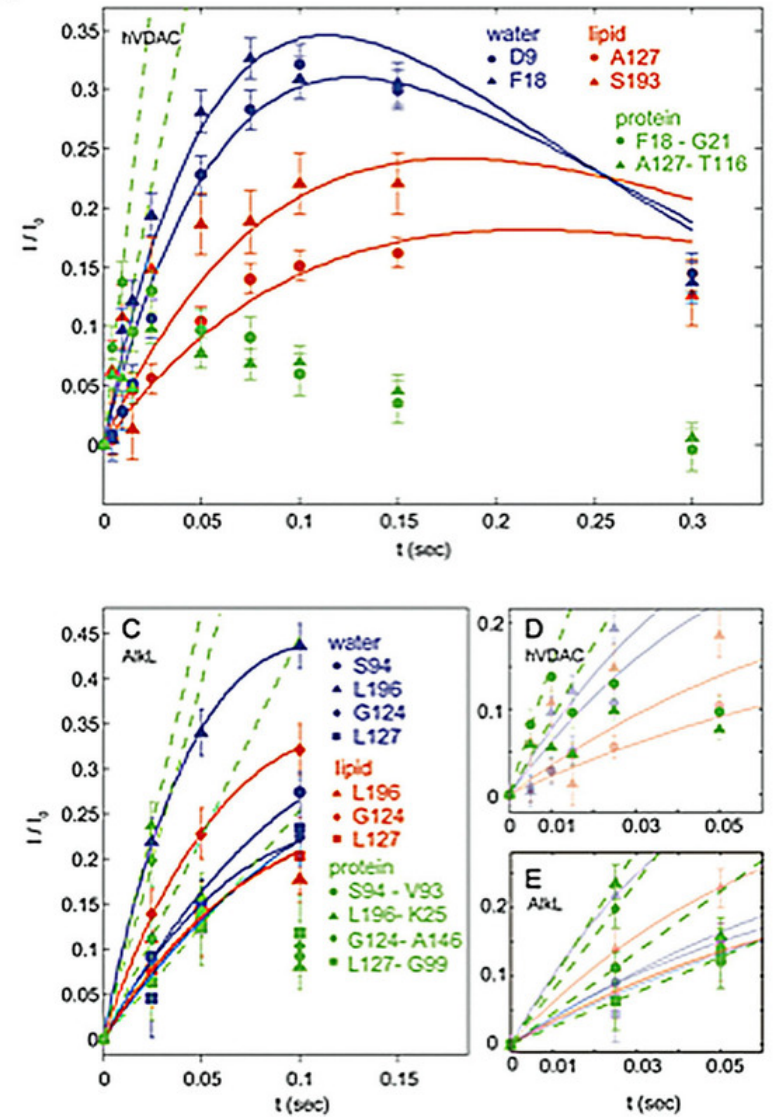

Figure 3: Evolution of magnetization in the HhNH spectrum of AlkL and hVDAC for selected residues. In A, the proton-proton planes from isolated residues are shown for 25, 50, and $100 \mathrm{~ms}$ in red, green, and black, respectively. The evolution of cross-peak intensities in hVDAC in B shows the water-protein signal intensity for two residues shielded from lipids, D9 and F18, as well as the lipid-protein signal intensity for two lipid exposed residues, A127 and S193. Curves were fit with the analytical function described in the text. In C, the evolution of isolated peaks in AlkL are shown. In D and E, the initial buildup of protein-protein peaks is used to estimate the rate of spin diffusion within the protein. The intensities are scaled to the protein amide signal at zero mixing time, an approximation of the initial source polarization. The decay of these diagonal intensities are shown in Figure S2. Error bars correspond to the root mean squared noise of the spectra.

and water-protein cross-peaks. This shows that spin diffusion within the protein is substantially suppressed by fast MAS and deuteration. Based on the relatively slow spin diffusion within the protein, a mixing time of $50 \mathrm{~ms}$ was chosen for both proteins and this value was used for the 4D spectra where more sites can be resolved. Site specificity is still imperfect under these conditions, since we do observe inter-residue transfer. However, as seen in Figure 3A, we typically only observe transfer to the nearest proton, for example, in the cross-strand beta sheets of hVDAC. In this topology, the cross-strand distance is usually below $3.0 \AA$, while the next closest proton is above $4.3 \AA$ away. For proton spin diffusion under fast MAS, or for proton driven spin diffusion, rate constants can be calculated from second order terms using perturbation or Floquet theory, and 
the transfer rate also becomes strongly chemical shift dependent. ${ }^{44,45}$ In this case, since spin diffusion and NOE are second order effects involving two couplings, their rates scale down with the sixth power of distance, and we would therefore expect about an order of magnitude reduction in the rate of transfer to other protons. This explains why we typically observe one inter-residue crosspeak in a beta sheet topology, and the specificity is expected to be within about $3 \AA$.

An initial rate approximation shows transfer rates of $3-15 \mathrm{~s}^{-1}$ within the protein (green dashed lines in Figure 3). For hVDAC, these estimates are reasonably reliable, since the initial points are below the maximum in the curve. For AlkL, this is not the case, and the fits underestimate the true rates. Intra-protein rates of up to $15 \mathrm{~s}^{-1}$ represents a 2-3 order of magnitude reduction in the deuterated sample at $55 \mathrm{kHz}$ as compared with protonated samples at $4-5 \mathrm{kHz}$ MAS. ${ }^{14,20,32}$ This comparison requires converting from the reported diffusion constants, $\mathrm{D}$, to transfer rates, which can be done applying the relation $\Omega=D / a^{2}$, where $D$ is the diffusion coefficient and a is the spacing between spins in a lattice model. ${ }^{14,32}$ A value of $\mathrm{D}$ of $0.8 \mathrm{~nm}^{2} / \mathrm{ms}$ was measured in rigid organic polymers. ${ }^{46} \mathrm{In}$ biological polymers, a lower value of $\mathrm{D}$ of 0.3 to $0.4 \mathrm{~nm}^{2} / \mathrm{ms}$ has been applied under similar conditions of low spinning rates. ${ }^{14,20,32}$ At a lattice spacing of 2 to $2.4 \AA$, a spin diffusion rate constant of $0.3 \mathrm{~nm}^{2} / \mathrm{ms}$, corresponds to a $5000-7500^{\mathrm{s}-1}$ transfer rate for this lower spinning frequency in fully protonated samples. In deuterated proteins, protons are spaced by at least $3 \AA$. A very rough approximation for the expected proton-proton transfer rate at $5 \mathrm{kHz}$ spinning can be calculated from a simple reduction by the 3rd power with distance, since the elementary transfer occurs via a first order process when the proton spectrum is broad and homogeneous due to ineffective averaging by MAS. This would result in an expected rate of about 2600-3800 ${ }^{s-1}$. Clearly, both the fast spinning rate, as well as the deuteration, contribute to quenching of the intraprotein transfer.

While at the lower spinning frequencies, evolution of magnetization during longitudinal mixing can be modeled based on Fick's laws of diffusion, ${ }^{47}$ the rates of spin diffusion with deuteration and fast MAS are reduced and highly inhomogeneous. Under such conditions, acquisition of 2D protonproton spectra and a matrix approach can be used to measure rates. ${ }^{11,48}$ When spin diffusion is fast within the protein, a simplification based on an overall scaling by a single global longitudinal relaxation time (T1) is sufficient to model the diffusive behavior and detect differences in overall exposure. ${ }^{12,20}$ With fast MAS and deuteration, we found that a single T1 did not fit the data and a more sophisticated model is needed. While a full rate matrix approach is clearly the gold standard for determination of cross-relaxation rates, protein resonances are woefully unresolved in 2D proton-proton spectra and improvement in resolution is afforded by filtering through a heteronucleus. This improvement in resolution in 3D spectra comes with the loss of the diagonal signals of the water and lipid species. We therefore chose to model the evolution of polarization, $\mathrm{M}(\mathrm{t})$, with several assumptions about the relaxation matrix, starting from the assumption that the lipids and water may be treated separately. Then,

$$
\begin{gathered}
\frac{\partial M_{p}(t)}{\partial t}=\left(-R_{1, p}-R_{m p}-R_{p p^{\prime}}\right) M_{p}+\left(R_{m p}\right) M_{m}+\left(R_{p p^{\prime}}\right) M_{p^{\prime}} \\
\frac{\partial M_{m}(t)}{\partial t}=\left(-R_{1, m}-\frac{1}{N} R_{m p}\right) M_{m}+\frac{1}{N}\left(R_{m p}\right) M_{p}
\end{gathered}
$$


Where the subscript $\mathrm{p}$ is protein, and $\mathrm{m}$ is for the mobile species, either lipid or water. R1,p is the longitudinal relaxation rate of protein, and the cross relaxation between mobile species and protein is $R_{w p}, R_{p p}$, refers to cross relaxation to all nearby protons of the protein. For simplicity, these other nearby protons are assumed to be in contact only with protein resonances. Lipids and water are assumed to equilibrate quickly among themselves, and $\mathrm{N}$ water or lipid protons are in contact with the amide resonance of the protein in fast equilibrium. Based on observed exponential decay of lipid signals, we can simplify to a single effective decay rate for lipid polarization, $\mathrm{R}_{1, \text { meff }}$

$$
\frac{\partial M_{m}(t)}{\partial t}=\left(-R_{1, m_{e f s}}\right) M_{m}
$$

We observed biexponential decay of water signal, and assume the protein is in contact primarily with the faster relaxing water population. This is consistent with observation of cross signals with only the broader water component in microcrystalline Crh samples under similar conditions. ${ }^{7}$ Then we can simplify the decay of water signal with a single rate, in the same way as for lipids.

We also chose to neglect magnetization transfer within the protein, since we observed moderate mixing of signals from $\mathrm{R}_{\mathrm{pp}}$, as indicated by the presence of relatively weak off-diagonal peaks in the amide region of the HhNH spectrum. We then find the solution,

$$
M_{p}(t)=M_{0, m} \frac{R_{m p}}{R_{1, p}+R_{m p}-R_{1, m_{e f f}}}\left(e^{-R_{1, m_{e f f}} t}-e^{-\left(R_{1, p}+R_{m p}\right) t}\right)
$$

We applied this analytical solution to extract approximate cross relaxation rates in hVDAC. The scaling factor $\mathrm{M}_{0, \mathrm{~m}}$ represents the initial polarization of the mobile species. The effective decay time for the lipids was set to the measured value of 5.3-5.5 ${ }^{\mathrm{s}-1}$. The effective decay time for water was allowed to vary between the two decay times observed in the biexponential decay, about $6^{\mathrm{s}-1}$. The fit lipid- and water-protein transfer rates were found to be similar, with values that ranged from 3 to $12^{s-1}$ for the isolated peaks of hVDAC and AlkL shown in Figure 3.

Since reliable fits require the correct scaling of the data, we took the initial protein amide peak intensity as an approximation for the initial lipid polarization. Due to differences in the recovery of lipids, water, and protein, this is estimated to introduce $10-20 \%$ error in the rates. To better focus on the lipids, we removed the influence of water by preparing a protein sample in D2O. Many amides in the membrane-spanning portion of the protein do not exchange with buffer, and their bulk $\mathrm{T} 1$ was found to be about 0.6 seconds, similar to the rate measured in $\mathrm{H} 2 \mathrm{O}$. Since this measurement includes decay due to cross-relaxation to lipids (T1 185 ms), we can safely assume that the protein T1 is longer than the apparent decay of 0.6 seconds. Under this assumption, the long-time decay of magnetization tracks the decay of lipid polarization, which may further stabilize the fits.

As further verification of these rates, we recorded hNHH spectra, placing the z-mixing at the end of the sequence. In such spectra, cross-relaxation rates can be determined by measuring both the decay of starting signal on the protein, as well as the buildup on lipids. ${ }^{16}$ Tracking the decay of starting polarization allows the determination of the total initial intensity and thereby provides a more direct measure of the rates, as in the short mixing approximation or the full matrix approach based on proton-proton spectra. ${ }^{11,48}$ Since imperfect water suppression severely affects hNHH spectra, we used the sample of hVDAC in D2O and measured the cross-relaxation from protein to lipids. This has the further advantage that the measurement takes place in isolation from transfer to water, and 
from any chemical exchange process, for example from water or from exchangeable side-chain protons. The average protein-lipid cross-relaxation rate using this approach was $6^{\mathrm{s}-1}$ (Figure S3). This rate is about an order of magnitude slower than the value of $90^{\mathrm{s}-1}$ reported for the average water-amide cross-relaxation in microcrystalline ubiquitin. ${ }^{16}$

Tracking the source polarization for rate determination is clearly preferred. However, this results in our case in detecting the water or lipid signal in the direct dimension, where there is loss of sensitivity due to imperfect solvent suppression and the resultant $\mathrm{t} 1$ noise. There was still some $\mathrm{t} 1$ noise evident near the lipid resonances in hNHH spectra of hVDAC in D2O. We therefore still expect there will be utility in our approach using $\mathrm{HhNH}$ spectra, although one must be cautious about the assumptions made for the water, lipid, and amide proton T1 relaxation times, as well as scaling the data by the initial protein signal which may introduce additional error if the initial polarization was not fully equilibrated. A more detailed investigation of cross-relaxation rates at different sites across the protein is left for the future.

Interestingly, analysis of average rates shows that cross relaxation to lipids or water can have a large influence on the measured longitudinal decay of amide proton magnetization. This can be appreciated by comparing the bulk decay of magnetization either after saturation of other protons, or starting with full signal on all protons. In hVDAC, the bulk $1 \mathrm{H} \mathrm{T1}$ of the amides as measured in these two ways is either $130 \mathrm{~ms}$ or $410 \mathrm{~ms}$, respectively. The faster decay is due to cross relaxation to lipids and water and implies an average cross-relaxation rate similar to the $6^{\mathrm{s}-1}$ determined for protein-lipid transfer. This observation has implications for the measurement of site-specific proton T1 for dynamics or accessibility studies suggesting that a detailed analysis of the intrinsic protein T1 must consider the effects of homonuclear magnetization transfer to water or lipids. On the other hand, solvent mediated PRE effects in samples with dissolved paramagnetic species still appear to highlight the exposed surface in accessibility studies; there was excellent agreement between the expected solvent exposed surface, and enhanced relaxation reported previously. ${ }^{49}$ The substantial cross-relaxation rates between protein and lipids or protein and water suggest that deuteration of water and lipids may be necessary to accurately measure site-specific proton T1 even with protein deuteration and very fast MAS.

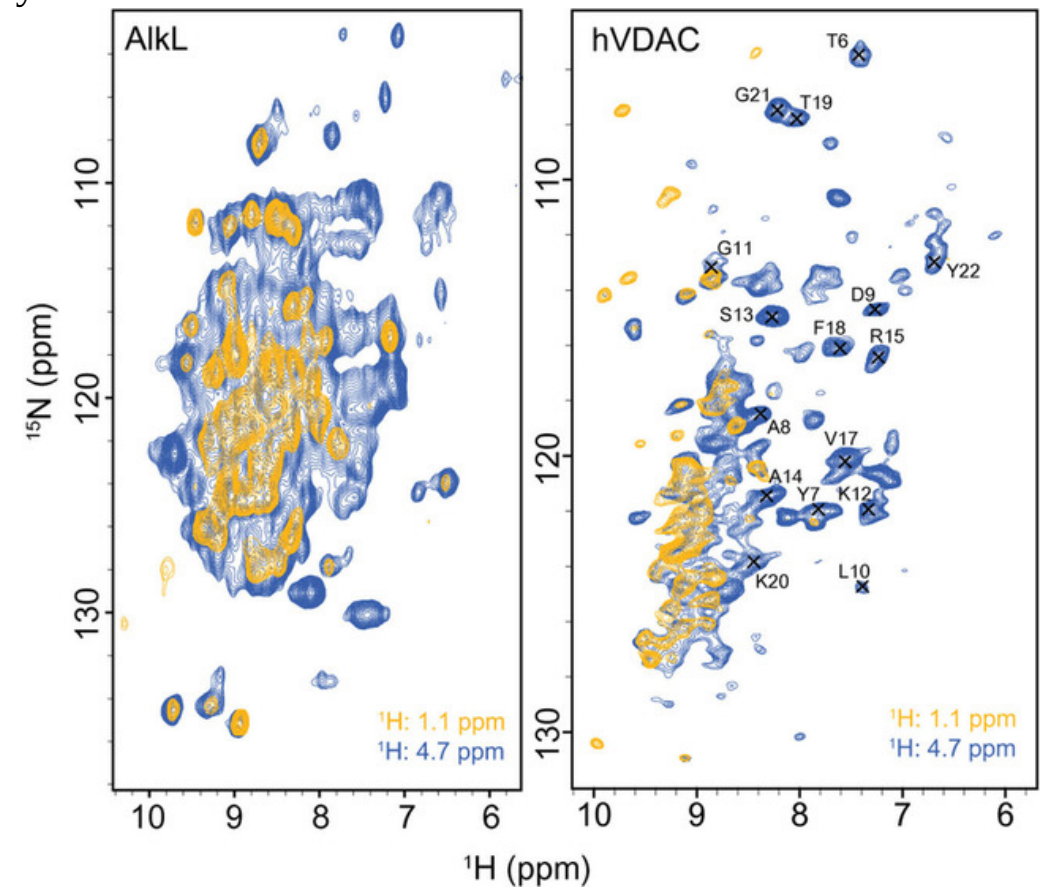

Figure 4: Lipid and water signal detected at the protein amides for AlkL and hVDAC. The 2D planes of the 3D HhNH spectrum are shown corresponding to the lipids (yellow, $1.1 \mathrm{ppm}$ ) and water (blue, $4.7 \mathrm{ppm}$ ). Assignments of the N-terminal helix are indicated for hVDAC. Proton-proton mixing proceeded for $50 \mathrm{~ms}$. 
Figure 4 shows 2D planes from the HhNH spectrum at the chemical shifts of both water (blue) and at the strongest signals of the lipid acyl chains (yellow). In hVDAC, although the amides are not fully resolved in an HN 2D plane, the helical residues of the N-terminus are well-separated from the more abundant beta sheet signals. Based on previously published assignments6 these helical residues can be identified unambiguously, with the exception of D16. All of these show strong correlations with water, and no cross-peak with lipids. This is fully in accordance with the known 19-stranded beta barrel structure of the channel determined in detergent micelles, ${ }^{36}$ as well as the placement of the helix within the large water-filled pore in crystals and in micelles. ${ }^{37,50,51}$ In total, 53 correlations with water and 42 with lipids can be observed, however only approximately 19 water correlations and 17 lipid correlations can be assigned with certainty due to extensive signal overlap in the HN plane.

Site-resolved correlations between the protein and the surrounding small molecules can be obtained in the HhnCANH spectrum. Figure 5 displays strip plots from spectra acquired on both AlkL and hVDAC showing correlations between representative protein residues and lipids and/or water. In the case of lipid correlations, two separate shifts are observable, one at $1.3 \mathrm{ppm}$ and one at $0.9 \mathrm{ppm}$, corresponding to the lipid $\mathrm{CH} 2$ and $\mathrm{CH} 3$ groups, respectively. For hVDAC we see 24 lipid correlations and 53 water correlations in total. Protein-lipid cross peaks tend to be weaker and therefore more difficult to observe. The fact that water-protein cross-peaks are stronger may be due to differences in mobility and structure, but are unlikely to arise from exchange pathways, considering that no significant correlation with exchangeable side-chains could be found. (Figure S1) Despite the reduced sensitivity, the clear advantage of the 4D spectrum is that assignments are almost completely unambiguous, due to the resolution afforded by 3 spectral dimensions, 13CA, $15 \mathrm{~N}$, and $1 \mathrm{H}$.

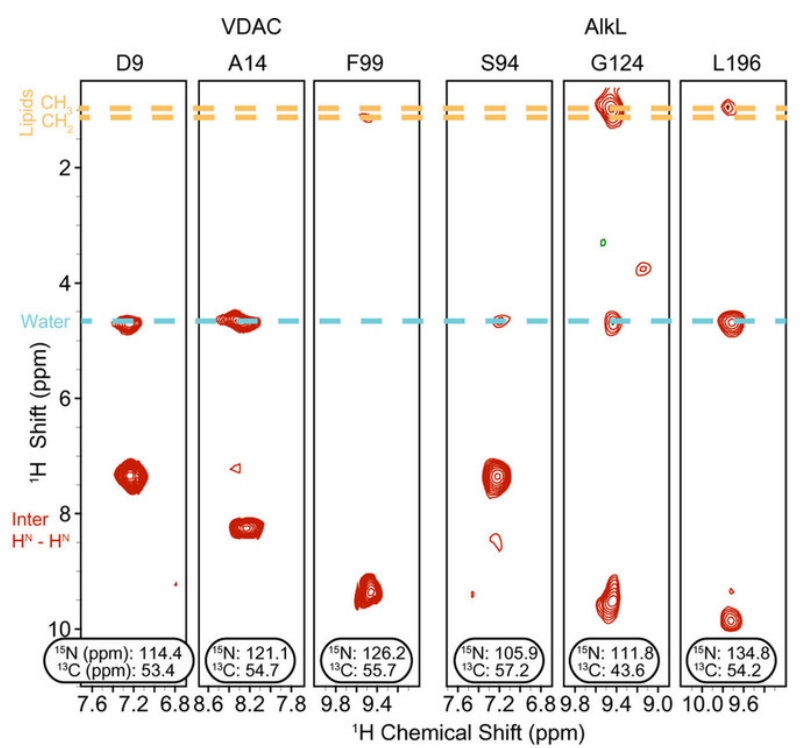

Figure 5: Selected strips from the 4D spectra of hVDAC and AlkL using $50 \mathrm{~ms}$ proton-proton mixing. The position of water and lipids are indicated by blue and yellow dashed lines, respectively. In most slices, only a single intense autocorrelation peak is observed in the amide region, indicating minimal magnetization transfer within the protein.

Figure 6 shows water- and lipid-protein correlations mapped onto the OmpW homology model of AlkL, generated with the swissmodel server ${ }^{52}$ and the solution NMR structure of hVDAC. ${ }^{51}$ For the residue mapping we combined information obtained from the well-resolved HhnCANH and the high-sensitivity HhNH experiments and used contacts from both spectra. Homologs of AlkL ${ }^{53-56}$ are composed of a transmembrane beta barrel, with long extracellular loops that are either flexible, or form a structured beta barrel extension, depending on the sample conditions, and the homolog. While the transmembrane beta barrel of homologous proteins is embedded in lipids, it is unclear from homology if the extracellular loops are in contact with lipids, are flexible, or are structured. At 
mixing times of $50 \mathrm{~ms}$, we observed only water contacts from the extracellular loops, while the transmembrane beta barrel showed efficient contact with both the lipids and water. This is in agreement with the homology model based on OmpW, where the lower barrel is embedded in the membrane, but the extracellular loops form a barrel extension that is water-exposed. On the other hand, at longer mixing times of $100 \mathrm{~ms}$, residues of the loops also show correlations with lipids. Further structural analysis of AlkL, as well as a more detailed investigation of rates would be needed to understand the flexibility of loop residues, and their impact on lipid-protein transfer.
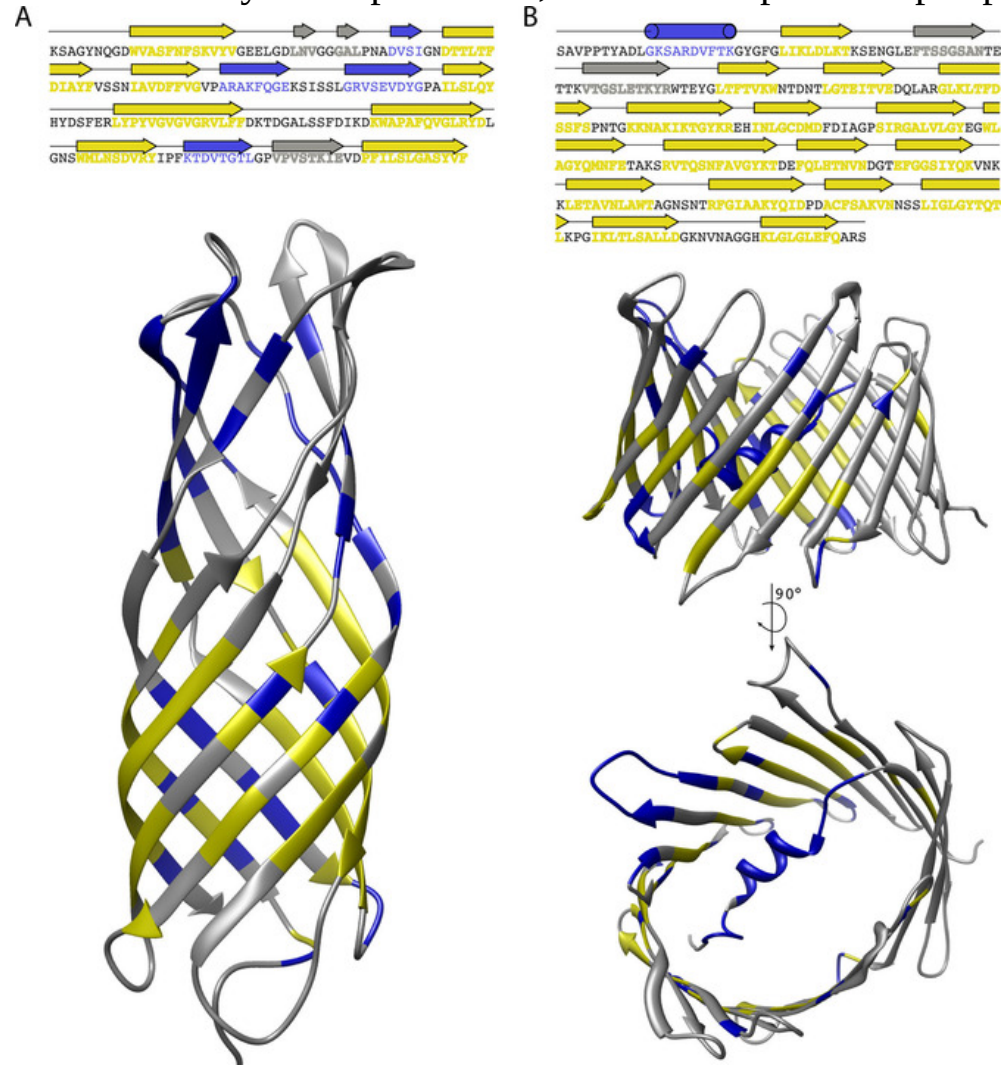

Figure 6: Water and lipid contacts shown on the homology model of AlkL using OmpW (PDB 2F1T) as a template (A) and the solution NMR structure (PDB 5JDP) of hVDAC (B). All residues that show a lipid contact are colored yellow, while residues that show only water contact are colored blue, and residues for which no contact could be observed or assigned are colored in grey. The topology (by homology in A) and sequence are indicated above each structure.

For hVDAC, combination of both 3D and 4D data (Figure 6) provides a more complete picture of water and lipid accessibility. As already seen in the 3D data, in hVDAC, the helical residues are clearly water-exposed and do not show any correlations to lipids. This is in accordance with the known structure of the protein, as the $\mathrm{N}$-terminal $\alpha$-helix is positioned horizontally in a large, waterfilled pore. Correlations to water can also be observed in the $\beta$-barrel, both in loop regions, and also with some residues in $\beta$-strands. Such loop residues with polar sidechains are energetically favored outside the membrane, but reside close to the lipids. That we do not observe lipid signal for some loop residues is therefore a testament to the high degree of selectivity possible under fast MAS, high magnetic field, and with deuteration. Protein-water proximities in $\beta$-strands are also not surprising, due to the barrel's large, water-filled pore. Lipid-protein contacts are only observed for residues in $\beta$-strands as well as in three loop residues, close to the end of $\beta$-strands (W64, Y146 and S240).

Insertion of membrane proteins in lipid bilayers has also been investigated with paramagnetic relaxation enhancement (PRE) measurements in solution. ${ }^{57}$ Whereas PRE has also been used for defining protein-protein ${ }^{49}$ or water-protein ${ }^{58}$ contacts in microcrystals, its potential for membrane 
insertion has not been exploited in solids. A drawback of the PRE technique is its indirect nature, as protein-lipid or protein-water contacts are not observed, but instead inferred due to an enhancement in relaxation where the solvent comes into contact with the protein. The method also may be less effective in larger, water accessible channels, such as hVDAC, since relaxation in the transmembrane part of the protein could be enhanced from the pore.

In conclusion, we have found that the combination of fast MAS, high magnetic field, and protein perdeuteration allows site specific detection of mobile species at the protein surface. We used the exchangeable amide proton as the detection nucleus, however, we anticipate that this methodology will be implemented in the future with different labeling schemes, for example, with methyl, or alpha proton labeling. The site specificity, as well as discrimination of water and lipid signals in a single spectrum, was used to probe the insertion of membrane proteins in lipid bilayers. We anticipate that the method presented here will strengthen the role of solid-state NMR in structural biology. It can be extended in a straightforward manner to test the influence of lipid compositions, membrane curvature and protein aggregation, thus allowing an in-depth characterization of membrane proteins that cannot easily be studied by other techniques.

\section{Acknowledgements}

We acknowledge financial support from the MPI for Biophysical Chemistry, and from the Deutsche Forschungsgemeinschaft (Emmy Noether program grant AN1316/1-1, SFB803 grant INST 186/794-3). T. Schwarzer was supported by the German Federal Ministry of Education and Research, Grant-No.031A178. T. Schubeis and G. Pintacuda received funding from the European Research Council (ERC-2015-CoG GA 648974).

References

[1] T. A. Cross, M. Sharma, M. Yi, H. X. Zhou, Trends Biochem. Sci. 2011, 36 , $117-125$.

[2] L. Frey, N. A. Lakomek, R. Riek, S. Bibow, Angew. Chem. Int. Ed. Engl.

2017, 56, 380-383.

[3] V. Kurauskas, A. Hessel, P. X. Ma, P. Lunetti, K. Weinhaupl, L. Imbert, B.

Brutscher, M. S. King, R. Sounier, V. Dolce, E. R. S. Kunji, L. Capobianco, C.

Chipot, F. Dehez, B. Bersch, P. Schanda, J. Phys. Chem. Lett. 2018, 9, 933-

938.

[4] O. Saurel, I. lordanov, G. Nars, P. Demange, T. Le Marchand, L. B.

Andreas, G. Pintacuda, A. Milon, J. Am. Chem. Soc. 2017, 139, 15901597.

[5] J. S. Retel, A. J. Nieuwkoop, M. Hiller, V. A. Higman, E. Barbet-Massin, J.

Stanek, L. B. Andreas, W. T. Franks, B. J. van Rossum, K. R. Vinothkumar,

L. Handel, G. G. de Palma, B. Bardiaux, G. Pintacuda, L. Emsley, W.

Kuhlbrandt, H. Oschkinat, Nat. Commun. 2017, 8, 2073.

[6] M. T. Eddy, Y. Su, R. Silvers, L. Andreas, L. Clark, G. Wagner, G. Pintacuda,

L. Emsley, R. G. Griffin, J. Biomol. NMR. 2015, 61, 299-310.

[7] A. Bockmann, C. Gardiennet, R. Verel, A. Hunkeler, A. Loquet, G.

Pintacuda, L. Emsley, B. H. Meier, A. Lesage, J. Biomol. NMR. 2009, 45, 319-327.

[8] A. Lesage, C. Gardiennet, A. Loquet, R. Verel, G. Pintacuda, L. Emsley, B. H. Meier, A. Bockmann, Angew. Chem. Int. Ed. Engl. 2008, 47, 58515854.

[9] A. Lesage, A. Bockmann, J. Am. Chem. Soc. 2003, 125, 13336-13337.

[10] G. Comellas, L. R. Lemkau, D. H. Zhou, J. M. George, C. M. Rienstra, J. Am.

Chem. Soc. 2012, 134, 5090-5099.

[11] D. Huster, K. Arnold, K. Gawrisch, J. Phys. Chem. B. 1999, 103, 243-251.

[12] C. Ader, R. Schneider, K. Seidel, M. Etzkorn, S. Becker, M. Baldus, J. Am.

Chem. Soc. 2009, 131, 170-176.

[13] G. J. Gallagher, M. Hong, L. K. Thompson, Biochemistry. 2004, 43, 7899-

7906.

[14] D. Huster, X. L. Yao, M. Hong, J. Am. Chem. Soc. 2002, 124, 874-883.

[15] T. Wang, H. Jo, W. F. DeGrado, M. Hong, J. Am. Chem. Soc. 2017, 139,

6242-6252.

[16] E. K. Paulson, C. R. Morcombe, V. Gaponenko, B. Dancheck, R. A. Byrd, K. W. Zilm, J. Am. Chem. Soc. 2003, 125, 14222-14223.

[17] H. Van Melckebeke, P. Schanda, J. Gath, C. Wasmer, R. Verel, A. Lange,

B. H. Meier, A. Bockmann, J. Mol. Biol. 2011, 405, 765-772. 
[18] C. Fernandez, C. Hilty, G. Wider, K. Wuthrich, Proc. Natl. Acad. Sci. USA 2002, 99, 13533-13537.

[19] A. Lange, S. Luca, M. Baldus, J. Am. Chem. Soc. 2002, 124, 9704-9705.

[20] W. Luo, M. Hong, J. Am. Chem. Soc. 2010, 132, 2378-2384.

[21] Z. L. Jia, L. L. Zhang, Q. Chen, E. W. Hansen, J. Phys. Chem. A. 2008, 112, 1228-1233.

[22] M. J. Knight, A. L. Webber, A. J. Pell, P. Guerry, E. Barbet-Massin, I. Bertini, I. C. Felli, L. Gonnelli, R. Pierattelli, L. Emsley, A. Lesage, T. Herrmann, G. Pintacuda, Angew. Chem. Int. Ed. Engl. 2011, 50, 11697-11701.

[23] L. B. Andreas, K. Jaudzems, J. Stanek, D. Lalli, A. Bertarello, T. Le Marchand, D. Cala-De Paepe, S. Kotelovica, I. Akopjana, B. Knott, S.

Wegner, F. Engelke, A. Lesage, L. Emsley, K. Tars, T. Herrmann, G.

Pintacuda, Proc. Natl. Acad. Sci. USA 2016, 113, 9187-9192.

[24] D. H. Zhou, J. J. Shea, A. J. Nieuwkoop, W. T. Franks, B. J. Wylie, C.

Mullen, D. Sandoz, C. M. Rienstra, Angew. Chem. Int. Ed. Engl. 2007, 46,

8380-8383.

[25] R. Linser, M. Dasari, M. Hiller, V. Higman, U. Fink, J. M. Lopez del Amo, S.

Markovic, L. Handel, B. Kessler, P. Schmieder, D. Oesterhelt, H. Oschkinat,

B. Reif, Angew. Chem. Int. Ed. Engl. 2011, 50, 4508-4512.

[26] M. Huber, S. Hiller, P. Schanda, M. Ernst, A. Bockmann, R. Verel, B. H.

Meier, ChemPhysChem 2011, 12, 915-918.

[27] T. Kobayashi, K. Mao, P. Paluch, A. Nowak-Krol, J. Sniechowska, Y.

Nishiyama, D. T. Gryko, M. J. Potrzebowski, M. Pruski, Angew. Chem. Int.

Ed. Engl. 2013, 52, 14108-14111.

[28] N. P. Wickramasinghe, S. Parthasarathy, C. R. Jones, C. Bhardwaj, F. Long,

M. Kotecha, S. Mehboob, L. W. M. Fung, J. Past, A. Samoson, Y. Ishii, Nat.

Methods 2009, 6, 215-218.

[29] Y. Nishiyama, M. Malon, Y. Ishii, A. Ramamoorthy, J. Magn. Reson. 2014,

244, 1-5.

[30] L. B. Andreas, T. Le Marchand, K. Jaudzems, G. Pintacuda, J. Magn. Reson.

2015, 253, 36-49.

[31] W. Luo, M. Hong, Solid State Nucl. Magn. Reson. 2006, 29, 163-169.

[32] K. K. Kumashiro, K. Schmidt-Rohr, O. J. Murphy, K. L. Ouellette, W. A.

Cramer, L. K. Thompson, J. Am. Chem. Soc. 1998, 120, 5043-5051.

[33] T. S. Schwarzer, M. Hermann, S. Krishnan, F. C. Simmel, K. Castiglione,

Protein Expression Purif. 2017, 132, 171-181.

[34] M. G. Jain, D. Lalli, J. Stanek, C. Gowda, S. Prakash, T. S. Schwarzer, T.

Schubeis, K. Castiglione, L. B. Andreas, P. K. Madhu, G. Pintacuda, V.

Agarwal, J. Phys. Chem. Lett. 2017, 8, 2399-2405.

[35] T. J. Malia, G. Wagner, Biochemistry. 2007, 46, 514-525.

[36] S. Hiller, R. G. Garces, T. J. Malia, V. Y. Orekhov, M. Colombini, G. Wagner,

Science. 2008, 321, 1206-1210.

[37] M. Bayrhuber, T. Meins, M. Habeck, S. Becker, K. Giller, S. Villinger, C.

Vonrhein, C. Griesinger, M. Zweckstetter, K. Zeth, Proc. Natl. Acad. Sci.

USA 2008, 105, 15370-15375.

[38] M. T. Eddy, T. C. Ong, L. Clark, O. Teijido, P. C. van der Wel, R. Garces, G.

Wagner, T. K. Rostovtseva, R. G. Griffin, J. Am. Chem. Soc. 2012, 134,

6375-6387.

[39] M. Dolder, K. Zeth, P. Tittmann, H. Gross, W. Welte, T. Wallimann, J.

Struct. Biol. 1999, 127, 64-71.

[40] T. D. K. Goddard, D. G., SPARKY 3. 2008, University of California, San

Francisco.

[41] A. W. Hing, S. Vega, J. Schaefer, J. Magn. Reson. 1992, 96, 205-209.

[42] A. Lends, F. Ravotti, G. Zandomeneghi, A. Bockmann, M. Ernst, B. H.

Meier, J. Biomol. NMR. 2018, 72, 69-78.

[43] L. Emsley, G. Bodenhausen, J. Magn. Reson. 1992, 97, 135-148.

[44] M. Veshtort, R. G. Griffin, J. Chem. Phys. 2011, 135.

[45] J. J. Wittmann, V. Agarwal, J. Hellwagner, A. Lends, R. Cadalbert, B. H.

Meier, M. Ernst, J. Biomol. NMR. 2016, 66, 233-242.

[46] J. Clauss, K. Schmidt-Rohr, H. W. Spiess, Acta Polym. 1993, 44, 1-17.

[47] A. Fick, J. Membr. Sci. 1995, 100, 33-38.

[48] S. Macura, R. R. Ernst, Mol. Phys. 1980, 41, 95-117.

[49] C. Oster, S. Kosol, C. Hartlmuller, J. M. Lamley, D. luga, A. Oss, M. L. Org,

K. Vanatalu, A. Samoson, T. Madl, J. R. Lewandowski, J. Am. Chem. Soc. 2017, 139, 12165-12174.

[50] R. Ujwal, D. Cascio, J. P. Colletier, S. Faham, J. Zhang, L. Toro, P. Ping, J.

Abramson, Proc. Natl. Acad. Sci. USA 2008, 105, 17742-17747.

[51] M. Jaremko, L. Jaremko, S. Villinger, C. D. Schmidt, C. Griesinger, S.

Becker, M. Zweckstetter, Angew. Chem. Int. Ed. Engl. 2016, 55, 10518-10521

[52] A. Waterhouse, M. Bertoni, S. Bienert, G. Studer, G. Tauriello, R.Gumienny, F. T. Heer, T. A. P. de Beer, C. Rempfer, L. Bordoli, R. Lepore, T. Schwede, Nucleic Acids Res. 2018, 46, W296-W303.

[53] I. Kucharska, P. Seelheim, T. Edrington, B. Liang, L. K. Tamm, Structure.2015, 23, 2234-2245.[54] R. Horst, P. Stanczak, K.

Wuthrich, Structure. 2014, 22, 1204-1209.

[55] D. S. Touw, D. R. Patel, B. van den Berg, PLoS One. 2010, 5, e15016.[56] H. Hong, D. R. Patel, L. K. Tamm, B. van den Berg, J.

Biol. Chem. 2006,281, 7568-7577.

[57] J. Dev, D. Park, Q. Fu, J. Chen, H. J. Ha, F. Ghantous, T. Herrmann, W.Chang, Z. Liu, G. Frey, M. S. Seaman, B. Chen, J. J. Chou,

Science. 2016,353, 172-175

[58] R. Linser, U. Fink, B. Reif, J. Am. Chem. Soc. 2009, 131, 13703-13708. 